\title{
Alimentación y cuidados paliativos en el paciente oncológico terminal
}

\author{
Nutrition and palliative care in terminal cancer patients \\ Alimentação e cuidados paliativos em pacientes oncológicos termináis
}

\author{
Mariana Vallejo Martínez ${ }^{1 *}$, Jeniffer Elizabeth Baque Hidalgo²
}

Recibido: 21 de enero de 2021. Aceptado para publicación: 1 de febrero de 2021.

https://doi.org/10.35454/rncm.v4n2.274

\begin{abstract}
Resumen
El paciente con cáncer avanzado y en etapa terminal es susceptible de cuidados paliativos de calidad. Esto se lleva a cabo cuando el profesional paliativista tiene amplio conocimiento de todas las esferas implicadas, como son la física, la psicológica, la emocional, la social y la espiritual. Los cuidados paliativos se realizan desde un enfoque multi e interdisciplinar, ofertando todos los servicios o cuidados posibles, esto incluye la alimentación. El servicio que pueden ofrecer los nutricionistas es muy prometedor y beneficioso a la hora de evaluar y tratar los síntomas de cada paciente. De esta forma se pueden definir esquemas apropiados al gusto y comodidad del paciente y sus familiares. La presente revisión tiene como objetivo abordar la alimentación oral en el contexto de los cuidados paliativos de los pacientes con cáncer en etapa terminal de la enfermedad.
\end{abstract}

Palabras clave: nutrición, cuidados paliativos, pacientes con cáncer, alimentación.

\section{Summary}

Patients with advanced and end-stage cancer are eligible for quality palliative care. This is possible when the palliative care professional demonstrated extensive knowledge about every domain involved, such as physical, psychological, emotional, social, and spiritual. Palliative care is delivered via a multi and interdisciplinary approach, offering every care service available, including nutrition. The support nutritionists can provide is very promising and beneficial when it comes to assessing and treating specific patient symptoms. In this way, appropriate treatment plans adapted to the preferences and comfort of the patients and their families can be defined. The objective of this review is to address oral feeding in the context of palliative care in patients with terminal cancer.

Keywords: Nutrition; Palliative Care; Cancer Patients; Feeding.

\section{Resumo}

O paciente com câncer avançado e em estágio terminal é suscetível de cuidados paliativos de qualidade. Isso é realizado quando o profissional paliativista possui amplo conhecimento de todas as esferas envolvidas como são a física, psicológica, emocional, social e espiritual. Os cuidados paliativos são realizados a partir de uma abordagem multi e interdisciplinar, oferecendo todos os serviços ou cuidados possíveis incluindo a alimentação. O serviço que os nutricionistas podem oferecer é muito promissor e benéfico na avaliação e tratamento dos sintomas de cada paciente. Desta forma, podem-se definir esquemas apropriados de acordo com o gosto e conforto do paciente e de suas famílias. A presente revisão tem como objetivo abordar a alimentação oral no contexto dos cuidados paliativos dos pacientes com câncer em estágio terminal.

Palavras-chave: nutrição, cuidados paliativos, pacientes com câncer; alimentação.
Servicio de Dolor y Paliativos del Instituto Oncológico Nacional “Dr. Juan Tanca Marengo". Solca, Guayaquil, Ecuador.
2 Posgrado de Cuidados Paliativos, Universidad Católica Santiago de Guayaquil, Ecuador.

*Correspondencia: Mariana Vallejo Martínez. mcvallejom@hotmail.es 


\section{INTRODUCCIÓN}

Hace varias décadas se ha comenzado a hacer visible el concepto de los cuidados paliativos (CP) o cuidados al final de la vida, sobre todo en las personas que se enfrentan a enfermedades incurables y que les queda (no siempre) poco tiempo de vida ${ }^{(1)}$. Aunque el pronóstico no permita prever la cura del paciente, siempre es posible mejorar la calidad de vida; por consiguiente, al paciente se le facilitan cuidados y servicios para que su estancia sea más placentera y vivir de la forma más activa, logrando inclusive despedirse de sus familiares, acciones que son necesarias y fundamentales para la paz interior.

A pesar de los avances en el conocimiento del cáncer $\mathrm{y}$ de la disponibilidad de nuevos tratamientos, aún hay una parte importante de pacientes con cáncer que no tendrán mejoría. Estos pacientes necesitan de cuidados paliativos para prolongar la sobrevida y mejorar la calidad de vida. En esta etapa, en ocasiones se mezclan tratamientos curativos y paliativos, los cuales no son mutuamente excluyentes. En las etapas más avanzadas, los cuidados paliativos tienen mayor énfasis, y mejorar la calidad de vida es el principal objetivo ${ }^{(1)}$.

La nutrición hace parte de los cuidados curativos y paliativos. Los pacientes con cáncer por la enfermedad misma y el tratamiento presentan alteraciones nutricionales que requieren de atención especial ${ }^{(2)}$. En esta revisión nos enfocaremos en el manejo de la alimentación en la etapa terminal del paciente con cáncer. Esta etapa presenta desafíos importantes para el equipo médico, el cuidador y la familia. La alimentación es uno de estos desafíos, que implica consideraciones tanto médico y biológicas como éticas.

\section{ALIMENTACIÓN Y CUIDADOS PALIATIVOS}

Los CP para los pacientes con cáncer avanzados se deben proporcionar por equipos multidisciplinarios en consulta hospitalaria o domiciliaria, y bajo pautas basadas en la evidencia científica ${ }^{(3)}$. Los CP se organizan en programas destinados a mantener o mejorar las condiciones de vida de los pacientes, cuyas enfermedades no responden por más tiempo al tratamiento curativo. Los CP intentan controlar no solo el dolor, los síntomas respiratorios, la fatiga, las alteraciones del sueño, las náuseas o la constipación, sino también el sufrimiento, para conseguir que estos enfermos vivan con plenitud sus últimos meses o días.

Las bases de los CP son la comunicación eficaz, el control de síntomas, el alivio del sufrimiento y el apoyo a la familia en el marco de un trabajo en equipo, cuyo objetivo principal es conseguir y mantener la mejor calidad de vida posible para el paciente, independiente de la respuesta de la enfermedad a un determinado tratamiento. En este contexto, la alimentación y el estado nutricional son esenciales; sin embargo, es importante tener claro ciertos conceptos y fases de la enfermedad para evaluar la respuesta, y no generar más ansiedad al paciente y su familia cuando se habla de nutrición ${ }^{(4)}$.

Para abordar adecuadamente el papel de la alimentación en el paciente con cáncer avanzado hay que diferenciar dos grupos de pacientes:

- El paciente en etapa terminal es aquel a quien se le ha agotado los tratamientos oncológicos disponibles, con una esperanza de vida de semanas o meses, y que presenta síntomas diversos que pueden variar en intensidad

- El paciente en fase de agonía es aquel que presenta una supervivencia limitada a días o pocas semanas.

Se hace énfasis en estas dos fases, ya que la alimentación cumple su función en ciertos pacientes incurables, pero no en los que se encuentran en fase de agonía.

\section{ALTERACIONES DEL ESTADO NUTRICIONAL DEL PACIENTE CON CÁNCER EN CUIDADOS PALIATIVOS}

La caquexia o progresiva pérdida involuntaria de peso en la consunción por cáncer conforma un síndrome complejo y multifactorial, que se desarrolla a través de diversos mecanismos, entre ellos, la anorexia ${ }^{(5,6)}$. Esta última, con la consiguiente pérdida de peso (síndrome de anorexia-caquexia), son frecuentes en el ámbito de los CP. Se estima que su incidencia oscila entre $15 \%$ y $40 \%$ en el momento del diagnóstico del cáncer, y aumenta hasta el $80 \%$ en los casos de enfermedad avanzada $^{(5)}$. Los pacientes con cáncer tienen un riesgo particularmente alto de desnutrición, porque tanto la enfermedad como sus tratamientos amenazan su estado nutricional. Se estima que las muertes del $10 \%$ $20 \%$ de los pacientes con cáncer se pueden atribuir a la desnutrición más que a la malignidad en si $^{(6)}$.

El síndrome de anorexia-caquexia es un complejo sintomático, que incluye anorexia, pérdida de peso, saciedad precoz, anemia, astenia, consunción tisular y disfunción de órganos, que con frecuencia se observa en cáncer pancreático, gástrico o esofágico, y con menor frecuencia en cáncer de mama o linfomas ${ }^{(7,8)}$. Se caracteriza por pérdida significativa de la masa muscular magra y grasa, y es una importante fuente de estrés psicológico 
para pacientes y familiares que enfrentan enfermedades que amenazan la vida ${ }^{(8)}$. Este síndrome se asocia con un peor pronóstico y peor calidad de vida de los pacientes. La caquexia afecta al $80 \%$ de los pacientes con cáncer terminal, y la agravación del estado nutricional puede deberse a la pérdida de proteínas en el intestino, diarrea o al hipercatabolismo ${ }^{(8)}$. La Figura 1 muestra la etiología de la caquexia en el paciente con cáncer.

La caquexia oncológica principalmente ocurre por inflamación sistémica, resistencia a la insulina, mitocondrias disfuncionales, estrés oxidativo y una mayor activación del sistema ubiquitina-proteasoma (UP) y autofagia $^{(9)}$. Las alteraciones metabólicas están mediadas por citocinas generadas por el tumor o el huésped, como el factor de necrosis tumoral (TNF) o la interleucina $6(\text { IL-6 })^{(10)}$. La activación de las vías UP y la autofagia explican, en gran parte, la importante pérdida de masa muscular esquelética. La reducción de la lipoproteína lipasa y el incremento de la lipólisis causan una pérdida de tejido adiposo y una elevación de los triglicéridos circulantes. También se produce un aumento de la gluconeogénesis y una reducción de la producción de la insulina, que pueden causar hiperglucemia. El tratamiento antitumoral también desempeña un papel muy importante en la etiología de la caquexia.

La anamnesis y la exploración física son importantes para valorar el estado nutricional del paciente. La primera facilita información sobre la velocidad y magnitud de la pérdida de peso, síntomas de malabsorción, alergias alimentarias, náuseas, vómitos y problemas en la deglución, uso de fármacos, consumo de alcohol, entre otros. Durante la exploración física, el médico debe prestar atención a la piel (sequedad, atrofia), la queilosis, la glositis y otros síntomas de carencia vitamínica, la emaciación, la pérdida de fuerza muscular y el edema con fóvea.

Según Fearon, la caquexia se clasifica como precaquexia, caquexia y caquexia refractaria, según el porcentaje de pérdida de peso ${ }^{(11)}$. El criterio diagnóstico para la caquexia es la pérdida de peso superior al 5 $\%$, o la pérdida de peso superior al $2 \%$ en individuos que ya mostraban alteración del estado nutricional, según un índice de masa corporal (IMC) $<20 \mathrm{~kg} / \mathrm{m}^{2}$, o presencia de sarcopenia (pérdida de fuerza y masa muscular esquelética).

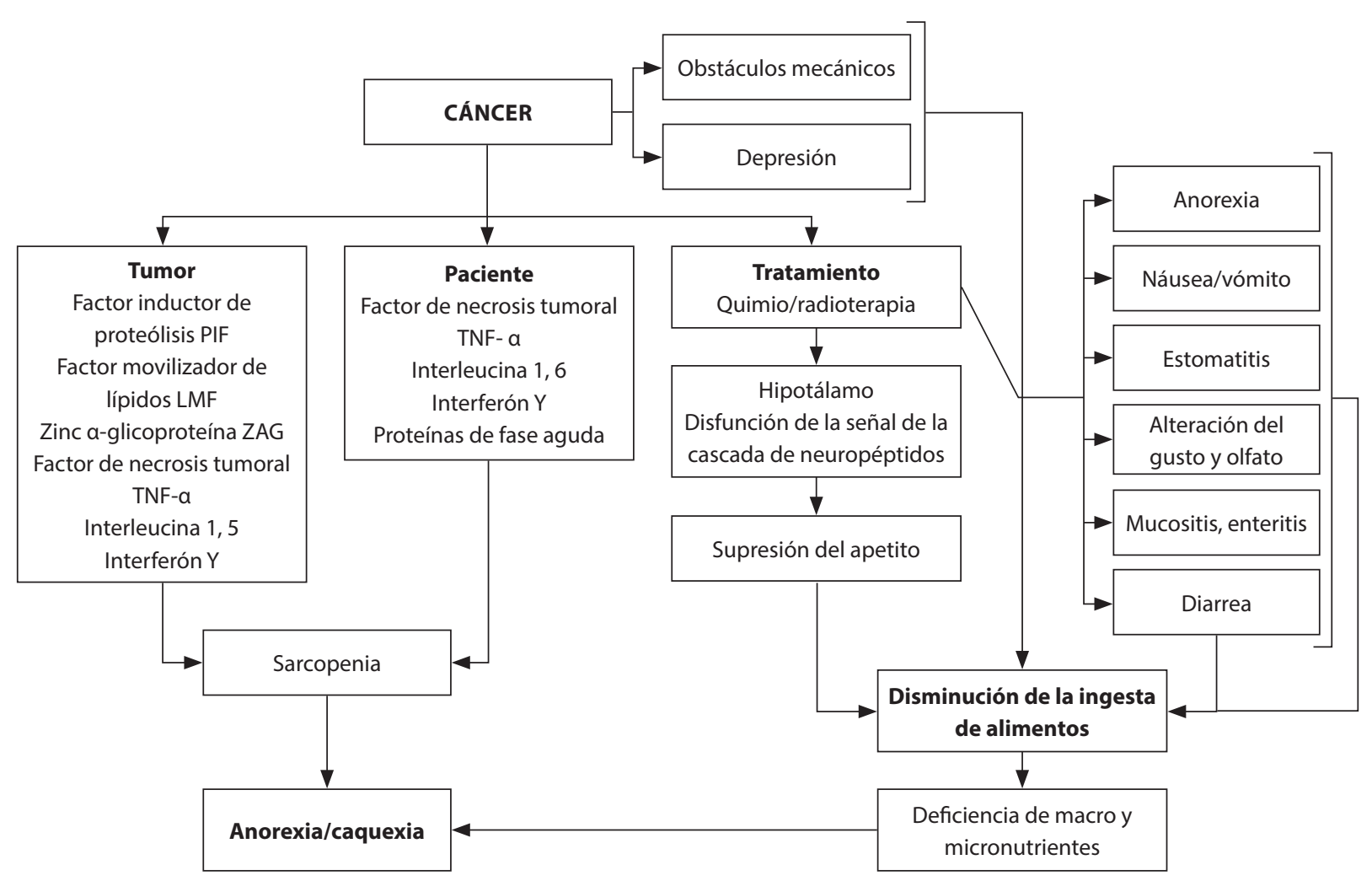

Figura 1. Génesis multifactorial de la caquexia inducida por el tumor. Tomado de ${ }^{(8)}$. 


\section{CAUSAS DE LOS TRASTORNOS DEL APETITO}

Las causas de los trastornos del apetito en los pacientes con cáncer avanzado son numerosas (Tabla 1) ${ }^{(12-14)}$. En todo enfermo es necesario realizar una adecuada evaluación que permita identificar las causas tratables, antes de aceptar fácilmente que se deba a un desinterés natural por comer o a una respuesta emocional ante la enfermedad.

Tabla 1. Anorexia en el paciente en fase terminal

\begin{tabular}{|c|c|}
\hline Etiología & $\begin{array}{l}\text { Posibilidades de } \\
\text { tratamiento }\end{array}$ \\
\hline $\begin{array}{l}\text { - Náuseas-vómito } \\
\text { - Comida poco apetitosa } \\
\text { - Presentación de grandes } \\
\text { porciones } \\
\text { - Saciedad temprana } \\
\text { - Deshidratación } \\
\text { - Estreñimiento } \\
\text { - Molestias bucales } \\
\text { - Disfagia } \\
\text { - Alteraciones bioquímicas } \\
\text { - Evolución de la } \\
\text { enfermedad } \\
\text { - Depresión } \\
\text { - Ansiedad }\end{array}$ & $\begin{array}{l}\text { - Antieméticos } \\
\text { - Posibilidad de selección } \\
\text { - Pequeñas porciones } \\
\text { - Tomar algo entre comidas } \\
\text { - Rehidratación oral } \\
\text { - Laxantes } \\
\text { - Cuidados de boca } \\
\text { - Cambiar consistencia de } \\
\text { dieta } \\
\text { - Corrección de anomalías, si } \\
\text { procede } \\
\text { - Probar corticoides } \\
\text { - Antidepresivos } \\
\text { - Ansiolíticos }\end{array}$ \\
\hline
\end{tabular}

Adaptado de ${ }^{(13)}$.

Algunos medicamentos causan alteración del gusto y del apetito. El gusto desempeña un papel crítico en el sentido general de bienestar y calidad de vida de las personas (Tabla 2). Las alteraciones del gusto pueden afectar negativamente el apetito y la ingesta de alimentos, lo que ocasiona deshidratación, pérdida de peso y desnutrición, y perjudica significativamente la calidad de vida y el estado de ánimo, por lo que es importante reconocerlos y valorar la posibilidad de retirarlos o modificarlos $^{(13)}$.

Tabla 2. Medicamentos que influyen negativamente en el gusto $y$ apetito

\begin{tabular}{|l|l|l|}
\hline Levodopa & Metronidazol & Aminofilina \\
\hline Litio & Captopril & Sulfonamidas \\
\hline Opioides & Fenitoína & Flurazepam \\
\hline Insulina & Anfetamina & 5-fluorouracilo \\
\hline $\begin{array}{l}\text { Compuestos de } \\
\text { hierro }\end{array}$ & AINE & Ciclofosfamida \\
\hline
\end{tabular}

AINE: antiinflamatorios no esteroideos. Adaptado de ${ }^{(14)}$.
A menudo, la pérdida del apetito resulta un elemento estresante. Los familiares se muestran preocupados por qué el paciente "no come lo suficiente", y este a su vez puede sentirse afectado también. De allí la importancia de mantener una buena comunicación con el paciente y la familia, y proporcionar la información adecuada. Es necesario hacer énfasis en que la pérdida de interés por la comida es un hecho natural en la proximidad de la muerte.

\section{ALIMENTACIÓN DEL PACIENTE EN ETAPA TERMINAL}

El abordaje nutricional de los pacientes con enfermedad oncológica avanzada y terminal está enfocado en la consejería nutricional. Algunos pacientes llegan a este estado recibiendo algún tipo de terapia médico-nutricional (TNM), cuyo objetivo principal es suplir los requerimientos nutricionales a través de suplementos nutricionales orales, la nutrición enteral o la nutrición parenteral. Sin embargo, es importante recalcar que no existe evidencia de que mejorar la ingesta nutricional en pacientes con cáncer avanzado en etapa terminal suponga una reducción en las comorbilidades, ni mayor sobrevida ${ }^{(15)}$. Si el paciente presenta caquexia refractaria, la administración de nutrientes, inclusive por vía artificial, no logrará mejorar su estado nutricional.

Las estrategias de nutrición en el paciente oncológico terminal deberán estar orientadas según la expectativa de vida, como se demuestra en el algoritmo de la Figura 2. La nutrición artificial no debe iniciarse si la esperanza de vida es menor de 3 meses. La decisión sobre iniciar o detener la nutrición artificial debe realizarse teniendo en cuenta el deseo del paciente y los cuestionamientos éticos que puedan surgir.

El equipo terapéutico paliativista debe elaborar estrategias específicas para mejorar la alimentación en el paciente con enfermedad avanzada terminal (Tabla 3). Según Beijer, la alimentación de confort se refiere a aquella que tiene como objetivo "hacer que el paciente se sienta cómodo física, social, emocional y espiritualmente" ${ }^{(17)}$. Este tipo de alimentación tiene como objetivo proporcionar la mejor calidad de vida posible y aliviar los síntomas. Para ello se recomiendan alimentos que el paciente pueda tolerar, y se ajusten a la forma en que el paciente enfrenta la progresión de la enfermedad. Son permitidos una baja ingesta o aportes nutricionales inadecuados. No se busca mantener un adecuado estado nutricional, se busca confort y placer para el paciente. La 
nutrición artificial en esta fase puede ser desproporcionada y causar más daño que beneficio.

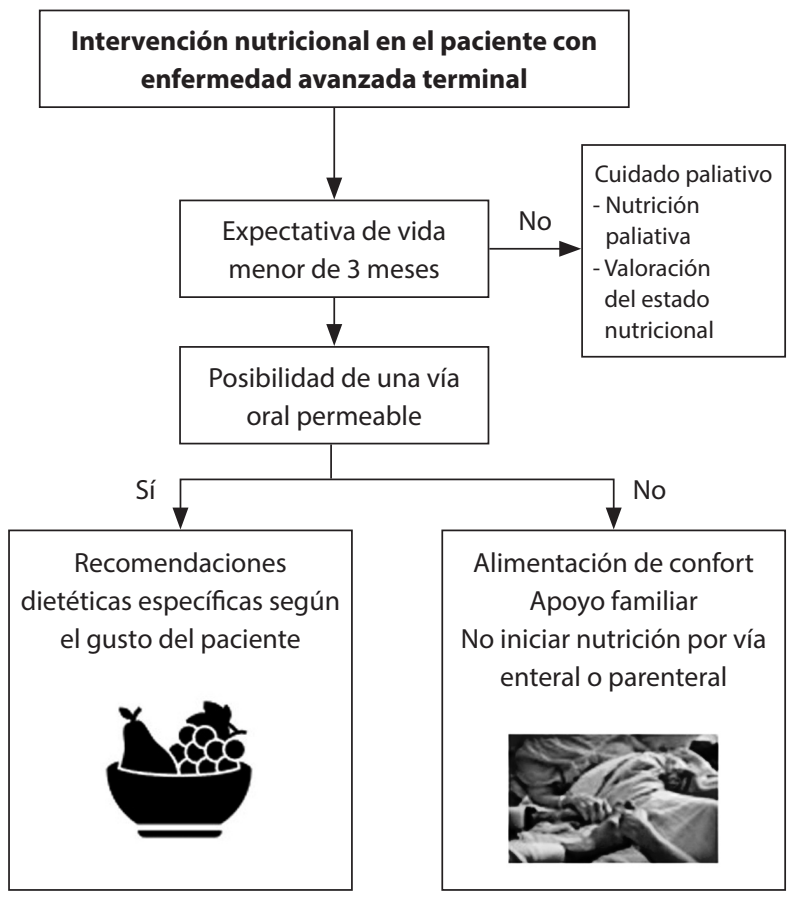

Figura 2. Algoritmo de la intervención nutricional en el paciente con enfermedad avanzada terminal. Tomado de ${ }^{(16)}$.
Un aspecto importante en el manejo de la alimentación de los pacientes con cáncer en etapa terminal es descartar causas reversibles de anorexia, como:

- Estreñimiento, náuseas, molestias en la boca, dolor, trastornos electrolíticos, depresión.

- Las alteraciones del gusto pueden abordarse reduciendo el contenido de urea de la dieta (menos carnes rojas), y marinando y condimentando los alimentos para enmascarar la percepción de sabores desagradables.

- Servir los alimentos a temperatura ambiente (no caliente) y animar al paciente a ingerir líquidos con las comidas.

- A los pacientes con anorexia se les debe permitir comer menos, pero con mayor frecuencia.

- Usar platos de tamaño pequeño, los cuales favorecen el consumo de porciones menores.

- El paciente debe implicarse en la selección del menú.

- El alimento debe proporcionarse siempre que el paciente tenga hambre.

- Para las comidas regulares es conveniente que el paciente se vista y se siente en la mesa, si es posible.

- Los pacientes que tienen anorexia pueden tomar estimuladores del apetito.

- El mejor estimulante es el propio gusto del paciente, que en ocasiones puede permitirse tomar un vaso de vino.

Tabla 3. Estrategias específicas para mejorar la alimentación en el paciente con enfermedad avanzada terminal

\begin{tabular}{|c|c|c|c|}
\hline Preparación del ambiente & $\begin{array}{c}\text { Presentación y preparación } \\
\text { de las comidas }\end{array}$ & Horario de las comidas & $\begin{array}{c}\text { Preparación de la vajilla y } \\
\text { cubertería }\end{array}$ \\
\hline $\begin{array}{l}\text { - Planificar las comidas como } \\
\text { ocasiones sociales, por } \\
\text { ejemplo, cumpleaños, visitas } \\
\text { - Crear un ambiente } \\
\text { placentero con flores, olores } \\
\text { a café } \\
\text { - Mostrar actitudes amables y } \\
\text { positivas } \\
\text { - Ambiente ventilado } \\
\text { tranquilizador con música } \\
\text { suave relajante }\end{array}$ & $\begin{array}{l}\text { - Color atractivo, olor, textura } \\
\text { suave de las comidas } \\
\text { - } 6 \text { a } 7 \text { porciones en raciones } \\
\text { reducidas } \\
\text { - Cada porción será servida de } \\
\text { forma separada y sin prisa, } \\
\text { debiendo retirar el plato } \\
\text { antes de servir el siguiente } \\
\text { - La cantidad de cada ingesta } \\
\text { se adaptará al enfermo sin } \\
\text { presiones } \\
\text { - Los pudines resultan de } \\
\text { entrada muy insípidas } \\
\text { - Ofrecer bebidas de colores } \\
\text { vivos } \\
\text { - Helados acompañados de } \\
\text { una cucharada de licor o una } \\
\text { salsa de frutas, lo que mejora } \\
\text { su sabor }\end{array}$ & $\begin{array}{l}\text { - Por lo general, es mejor por } \\
\text { las mañanas, y están en } \\
\text { condiciones de ingerir un } \\
\text { desayuno completo (esta } \\
\text { puede ser la única comida } \\
\text { normal en el proceso de la } \\
\text { enfermedad) } \\
\text { - } \quad \text { Debe existir flexibilidad } \\
\text { horaria } \\
\text { - No ofrecer sus comidas } \\
\text { favoritas mientras vomitan }\end{array}$ & $\begin{array}{l}\text { - Vajilla pequeña } \\
\text { - Tasas ligeras con asas } \\
\text { adecuadas para que puedan } \\
\text { ser sujetadas por dedos } \\
\text { - deformes o debilitados } \\
\text { - Pajitas flexibles } \\
\text { - Mangos de cubiertos anchos } \\
\text { - Copas para las bebidas o el } \\
\text { agua }\end{array}$ \\
\hline
\end{tabular}

Adaptado de ${ }^{(17,18)}$. 
El cuidado nutricional debe ir acompañado de actividad física y movilización del paciente ${ }^{(19)}$, los cuales deben adaptarse a la condición de cada paciente. Para contrarrestar la desnutrición en pacientes con cáncer avanzado existen pocos agentes farmacológicos con efectos limitados ${ }^{(15)}$.

El abordaje nutricional de estos pacientes debe realizarse siempre teniendo en cuenta el deseo y los gustos del paciente. De esta forma se respeta el principio ético de autonomía y la dignidad del paciente, tal y como lo estipula la Declaración de Cartagena sobre el derecho al cuidado nutricional y la lucha contra la desnutrición en su principio número $1^{(20)}$.

\section{AGENTES FARMACOLÓGICOS ESTIMULADORES DEL APETITO: OREXÍGENOS}

Se pueden considerar estimulantes del apetito aquellos medicamentos y tratamientos que contribuyan a mejorar la plurisintomatología, y como norma general se administrarán como prueba durante un período de $10 \mathrm{a}$ 14 días, interrumpiéndose el tratamiento en caso de no obtener efectos positivos $^{(21)}$. Se han desarrollado varios fármacos orexigénicos específicos para tratar la anorexia en pacientes con cáncer. Se ha demostrado que todos tienen alguna utilidad, pero ninguno modifica la enfermedad; por ejemplo, estudios recientes indican la posibilidad de que la gastroparesia sea la razón de la anorexia o náusea crónica en el cáncer avanzado. Al utilizar metoclopramida en infusión continua en dosis de $30-120 \mathrm{mg} / 24$ horas en 19 pacientes se consiguió mejoría en 14 de 16 (85\%), con aumento de la ingesta de calorías diarias ${ }^{(22)}$ (Tabla 4$)$.

\section{CONCLUSIONES}

La alimentación y el bienestar en la etapa terminal deben tener el propósito de proporcionar placer al paciente y mejorar su calidad de vida y de partida. La alimentación tiene un componente afectivo, cultural y social innegable, por lo que se debe adecuar a cada enfermo en particular. El abordaje interdisciplinario y la comunicación son pilares fundamentales para proponer los esquemas con la mejor comprensión de las necesidades reales del paciente y familia.

\section{Financiación}

El presente artículo no tuvo financiación.

\section{Conflicto de intereses}

Las autoras declaran no tener conflicto de interés.

\section{Declaración de la autoría}

MV diseñó el artículo. MV y JB escribieron y revisaron el artículo. MV validó la versión final del manuscrito.

Tabla 4. Medicamentos orexígenos

\begin{tabular}{|c|c|c|c|c|c|c|c|}
\hline $\begin{array}{l}\text { Esteroides } \\
\text { anabólicos }\end{array}$ & $\begin{array}{l}\text { Proges- } \\
\text { tágeno }\end{array}$ & Corticoides & $\begin{array}{l}\text { Estimulantes } \\
\text { de la motilidad } \\
\text { intestinal }\end{array}$ & Vitamina & $\begin{array}{l}\text { Canna- } \\
\text { binoide }\end{array}$ & $\begin{array}{c}\text { Antidepresivo } \\
y \\
\text { antipsicóticos }\end{array}$ & Otros \\
\hline $\begin{array}{l}\text { Deca- } \\
\text { durabolin } \\
100 \mathrm{mg}\end{array}$ & $\begin{array}{l}\text { Acetato de } \\
\text { megestrol: } \\
160-1600 \\
\mathrm{mg} / \text { día }\end{array}$ & $\begin{array}{l}\text { Dexametasona } \\
\text { 2-8 mg/día por } 1 \\
\text { semana } \\
\text { Metilprednisolona } \\
\text { 16-40 mg/día }\end{array}$ & $\begin{array}{l}\text { Metoclopramida } \\
30-120 \text { mg en } \\
24 \text { horas infusión } \\
\text { continua }\end{array}$ & $\begin{array}{l}\text { Zinc } 50-180 \\
\text { mg por día por } \\
\text { 1-2 semanas } \\
\text { Complejo B } \\
\text { 5-40 mg por } \\
\text { día }\end{array}$ & $\begin{array}{l}\text { Dronabinol } \\
5-7,5 \text { mg/ } \\
\text { día } 1 \text { hora } \\
\text { después de } \\
\text { las comidas }\end{array}$ & $\begin{array}{l}\text { Mirtazapina } \\
\text { 15-30 mg/día } \\
\text { Olanzapina } 5 \\
\text { mg/día }\end{array}$ & $\begin{array}{l}\text { Melatonina } 20 \\
\text { mg en la noche } \\
\text { Talidomida } \\
50-200 \text { mg } \\
\text { Pentoxifilina } 400 \\
\text { mg } 3 \text { veces por } \\
\text { semana }\end{array}$ \\
\hline
\end{tabular}

Adaptado de ${ }^{(22)}$.

\section{Referencias bibliográficas}

1. Palliative care [Internet]. World Health Organization. [Consultado en agosto de 2020]. Disponible en: http://www. who.int/cancer/palliative/definition/en/
2. Arends J, Bachmann P, Baracos V, Barthelemy N, Bertz H, Bozzetti $\mathrm{F}$, et al. ESPEN guidelines on nutrition in cancer patients. Clin Nutr. 2017;36(1):11-48. doi: 10.1016/j. clnu.2016.07.015. 
3. Ferrell BR, Temel JS, Temin S, Alesi ER, Balboni TA, Basch $\mathrm{EM}$, et al. Integration of palliative care into standard oncology care: American Society of Clinical Oncology Clinical Practice Guideline Update. J Clin Oncol. 2017;35(1):96-112. doi: 10.1200/JCO.2016.70.1474.

4. Camblor-Álvarez M, Ocón-Bretón MJ, Luengo-Pérez LM, Virizuela JA, Sendrós-Madroño MJ, Cervera-Peris M, et al. Soporte nutricional y nutrición parenteral en el paciente oncológico: informe de consenso de un grupo de expertos. Nutr Hosp. 2018;35(1):224-33. doi: 10.20960/nh.1361.

5. Rodríguez VD, Frías-Toral E, Santana PS. Diagnóstico nutricional en el paciente oncológico: importancia y herramientas para realizarla. Rev Oncol Ecu. 2018;28(3):169-80. doi: $10.33821 / 271$.

6. Roeland EJ, Bohlke K, Baracos VE, Bruera E, Del Fabbro E, Dixon S, et al. Management of cancer cachexia: ASCO Guideline. J Clin Oncol. 2020;38(21):2438-53. doi: 10.1200/ JCO.20.00611.

7. Arends J, Baracos V, Bertz H, Bozzetti F, Calder PC, Deutz NEP, et al. ESPEN expert group recommendations for action against cancer-related malnutrition. Clin Nutr. 2017;36(5):1187-96. doi: 10.1016/j.clnu.2017.06.017.

8. Rodríguez VD, Frías-Toral E, Santana PS. Requerimientos ajustados a la desnutrición del paciente oncológico. Rev Oncol Ecu. 2019;29(2):83-96. doi: 10.33821/92.

9. Zhang Y, Wang J, Wang X, Gao T, Tian H, Zhou D, et al. The autophagic-lysosomal and ubiquitin proteasome systems are simultaneously activated in the skeletal muscle of gastric cancer patients with cachexia. Am J Clin Nutr. 2020;111(3):5709. doi: 10.1093/ajcn/nqz347.

10. Miján de la Torre A. El músculo, elemento clave para la supervivencia en el enfermo neoplásico. Nutr Hosp. 2016;33(1):175. doi: $10.20960 /$ nh. 175 .

11. Fearon K, Strasser F, Anker SD, Bosaeus I, Bruera E, Fainsinger $\mathrm{RL}$, et al. Definition and classification of cancer cachexia: an international consensus. Lancet Oncol. 2011;12(5):489-95. doi: 10.1016/S1470-2045(10)70218-7.

12. Davis MP, Dreicer R, Walsh D, Lagman R, LeGrand SB. Appetite and cancer-associated anorexia: a review. J Clin Oncol. 2004;22(8):1510-7. doi: 10.1200/JCO.2004.03.103.
13. Medicamentos que causan trastornos del gusto [Internet]. Salud y Fármacos. [citado el 2 de noviembre de 2020]. Disponible en: http://www.saludyfarmacos.org/lang/es/ boletin-farmacos/boletines/ago201901/08_me

14. De Luca Monasterios FM, Roselló Llabrés X. Etiopatogenia y diagnóstico de la boca seca. Av Odontoestomatol 2014; 30( 3):121-128.Disponible en: http://scielo. isciii.es / scielo.php? script $=$ sci_arttext\&pid=S0213$12852014000300004 \& \operatorname{lng}=$ es.

15. Bozzetti F. Is there a place for nutrition in palliative care? Support Care Cancer. 2020;28(9):4069-75. doi: 10.1007/ s00520-020-05505-x.

16. Gómez C, Alonso A. Guía clínica de soporte nutricional en cuidados paliativos. SECPAL. 2015. Disponible en: http:// afam.org.ar/textos/22_06_17/guia_practica_de_soporte_ nutricional_en_cuidados_paliativos.pdf

17. Beijer S, Vogel J, Jager-Wittenaar H. Alternative terminology for the confusing term "palliative nutrition". Clin Nutr. 2017;36(6):1723-4. doi: 10.1016/j.clnu.2017.09.012.

18. Pérez-Camargoa DA, Allende-Péreza $S$, Domínguez-Ocadioa G, Verástegui-Avilésa E. Alimentación e hidratación en Medicina Paliativa. 2013;12(4):265-75.

19. Demark-Wahnefried W, Schmitz KH, Alfano CM, Bail JR, Goodwin PJ, Thomson CA, et al. Weight management and physical activity throughout the cancer care continuum. Cancer J Clin. 2018;68(1):64-89. doi: 10.3322/caac.21441.

20. Cárdenas D, Bermúdez C, Echeverri S, Pérez A, Puentes M, López L, et al. Declaración de Cartagena. Declaración Internacional sobre el Derecho al Cuidado Nutricional y la Lucha contra la Malnutrición. Nutr Hosp. 2019;36(4):97498. doi: 10.20960/nh.02701.

21. Ezeoke CC, Morley JE. Pathophysiology of anorexia in the cancer cachexia syndrome. J Cachexia Sarcopenia Muscle. 2015;6(4):287-302. doi: 10.1002/jcsm.12059.

22. Peñas R, Sorribes P. Tratamiento farmacológico de la anorexia y caquexia neoplásicas. SEOM, 2017. [citado 19 de enero de 2021]. Disponible en: http://www.seom.org/seomcms/images/stories/recursos/infopublico/publicaciones/soporteNutricional/pdf/cap_16.pdf 